\title{
The sutureless and rapid-deployment aortic valve replacement international registry: lessons learned from more than 4,500 patients
}

\author{
Marco Di Eusanio, Paolo Berretta \\ Cardiac Surgery Unit, Lancisi Cardiovascular Center, Polytechnic University of Marche, Ancona, Italy \\ Correspondence to: Paolo Berretta. Cardiac Surgery Unit, Lancisi Cardiovascular Center, Polytechnic University of Marche, Via Conca 71, 60126 Ancona, \\ Italy. Email: p.berretta@icloud.com.
}

\begin{abstract}
The treatment options for patients with aortic valve disease have considerably expanded over the last decade. The remarkable advances in catheter-based technology, the popularizing of minimally invasive (MI) surgery, and the introduction of new valve technologies, such as sutureless and rapid-deployment (SURD) valves have led to a paradigm shift in the management of aortic valve pathologies. Yet, given their recent introduction, the current evidence on sutureless and rapid-deployment aortic valve replacement (SURD-AVR) has been limited thus far. The Sutureless and Rapid-Deployment Aortic Valve Replacement International Registry (SURD-IR) was established in 2015 by a consortium of 18 research centers to assess safety, efficacy, shortand long-term outcomes of SURD-AVR interventions. The present keynote lecture aims to assess and comment on the real-world evidence for SURD-AVR surgery generated from the SURD-IR.
\end{abstract}

Keywords: Sutureless valve; rapid-deployment valve; aortic valve replacement (AVR); Sutureless and Rapid-Deployment Aortic Valve Replacement International Registry (SURD-IR)

Submitted Apr 06, 2020. Accepted for publication Apr 28, 2020.

doi: 10.21037/acs-2020-surd-21

View this article at: http://dx.doi.org/10.21037/acs-2020-surd-21

\section{Introduction}

The treatment options for patients with aortic valve disease have significantly expanded over recent years. The tremendous growth of transcatheter aortic valve implantation (TAVI) procedures, the increased popularity of less invasive approaches for surgical aortic valve replacement (SAVR) and the introduction of new valve technologies, such as sutureless and rapid-deployment (SURD) valves, have led to a paradigm shift in the management of aortic valve pathologies $(1,2)$.

The sutureless concept of aortic valve implantation was introduced in the early 1960s to simplify the implantation technique and shorten cardiopulmonary bypass (CPB) duration (3). However, this approach fell out of favor due to several drawbacks, including frequent valve-related thromboembolic complications and severe paravalvular leakages (4). More recently, with the advent of bovine pericardial valve prostheses, new SURD valve prostheses have been developed based on the modern experience with TAVI. Given the recent introduction and the short observational interval, the current literature on sutureless and rapid-deployment aortic valve replacement (SURDAVR) interventions is limited to small multicenter clinical trials or single center series, that do not adequately reflect the real-world situation and do not allow for a thorough evaluation of procedural and clinical results of SURD valve technologies. To overcome these limitations and provide more convincing evidence for SURD-AVR surgery, the Sutureless and Rapid-Deployment Aortic Valve Replacement International Registry (SURD-IR) was established in 2015 with the aim of evaluating safety, efficacy, short and long-term outcomes of SURD-AVR (5).

The present keynote lecture aims to assess and comment on the real-world evidence on SURD-AVR surgery, generated from the SURD-IR.

\section{SURD-IR design}

The SURD-IR is a multicenter retrospective and 


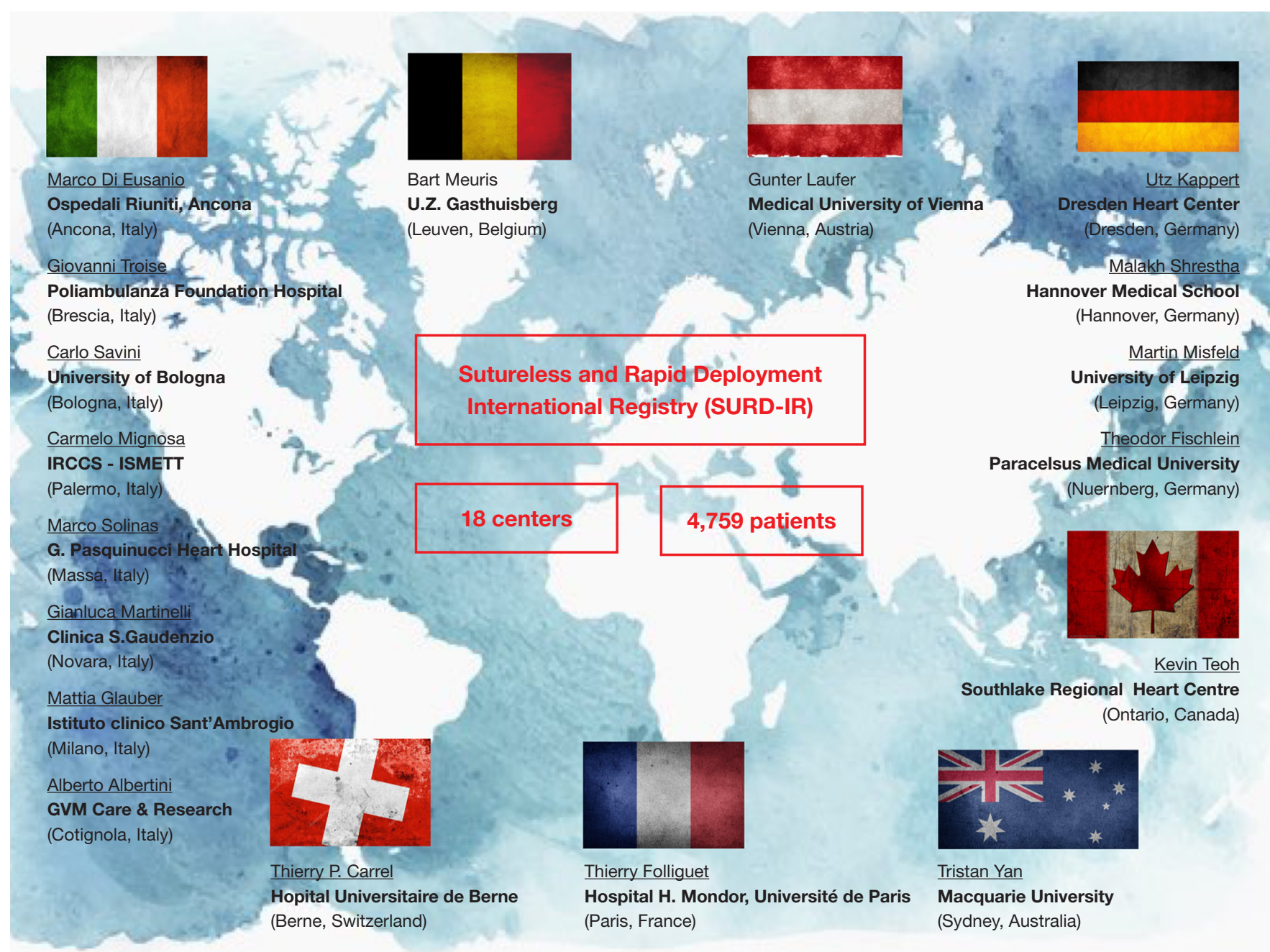

Figure 1 SURD-IR participating centers.

prospective registry founded by a consortium of research cardiac centers, namely the International Valvular Surgery Study Group (IVSSG) (5). Currently, patients are enrolled from 18 sites in Europe, Australia and Canada (Figure 1). The study population is defined as patients undergoing SURD-AVR intervention, using any available SURD valve prosthesis, either by conventional sternotomy or a less invasive approach. Valve prosthesis types included Perceval S (Livanova PLC, London, UK) EDWARDS INTUITY/ INTUITY Elite (Edwards Lifesciences, Irvine, California, USA) and Enable 3F (Medtronic, Minneapolis, USA). However, since the Enable $3 \mathrm{~F}$ valve was recalled from the market, patients who received this prosthesis were excluded from the registry.

Details of site selection and invitation have been previously published (5). Briefly, centers that had published reports on more than 50 SURD-AVR cases were initially invited to participate in the present database, as this was hypothesized to represent experienced centers with quality data collection. Further institutions recommended by the IVSSG Research Steering Committee were also invited to participate in the registry. Ethics approval was obtained at each site. Participating SURD-IR centers enrolled between 40 and 735 patients and collected information on patient demographics, comorbidities, functional status, imaging studies, surgical data, post-operative course, clinical and hemodynamic outcomes. Following electronic data submission, each dataset was evaluated to ensure that all patients were over 18 years old. All variables between datasets were assessed, with identical variables collated 
into a centralized database. Isolated variables reported by less than $25 \%$ of centers were excluded from analysis. Individually missing data and center-specific non-reported data were coded separately. Clinically important absent data were queried with the submitting center. Data were analyzed for clinical face validity and internal validity. Submitted clinical data were compared against published data for inconsistencies.

\section{End-points}

Over 190 variables were collected for each patient. Variables of interest for the SURD-IR involved: (I) clinical data variables, including age, sex, NYHA class, CCS class, comorbidities, indications for surgery, baseline echocardiographic and hemodynamic data and patient history; (II) risk assessment variables, including Logistic EuroSCORE, EuroSCORE II, STS PROM risk and major organ system compromises; (III) operative details, including surgical approach, concomitant procedures, type of prosthesis, prosthesis size and operative times; (IV) technical outcomes, including immediate procedural success (defined as successful first implant of the valve, not requiring repeated cross-clamping), occurrence of first implant failure, valve migration/embolization, conversion to sutured AVR, post-implantation aortic valve regurgitation and pressure valve gradients; (V) hospital outcomes, including mortality and cause of death, echocardiography and hemodynamic parameters, perioperative blood transfusion, postoperative complications (cardiac, renal, respiratory, neurologic, infective, gastrointestinal and wound complications), cardiac and aortic valve re-interventions and duration of ICU and hospital stay; (VI) follow up data, including mortality and cause of death, cardiac and/or neurological complications, echocardiography and hemodynamic data and occurrence and cause of aortic valve reintervention.

\section{Evidence from SURD-IR}

\section{Demographics and risk profile}

A total of 4,759 patients undergoing SURD-AVR over a 12-year period between 2007 and 2019 were enrolled in the SURD-IR. Of these, 123 (2.6\%) received an Enable $3 \mathrm{~F}$ valve and were excluded from the registry. The SURDIR study population consisted of near octogenarians (mean age 76 years, $32.8 \%>80$ years), with a considerable burden of comorbidities that translated into a median
Logistic EuroSCORE of $8.1 \%$ (IQR, 5.1-12.6\%). However, consistent with the current worldwide trends in conventional SAVR described in several national registries $(6,7)$, data from the SURD-IR revealed a change in patient characteristics, with a significant decrease in estimated surgical risk over time (8). This is likely due to both the exponential growth in TAVI case volumes that had the highest penetration in elderly and higher risk patients $(1,6,7)$ and the increasing adoption of biological valves in younger, lower risk patients $(9,10)$. Indeed, the overall mean age of the SURD-IR cohort decreased from 78.4 to 73.4 years over time. Accordingly, the rate of younger patients ( $<65$ years of age) who underwent SURD-AVR increased from $1.5 \%$ to $12.6 \%$ (8).

\section{Peculiar patient subgroups}

\section{Bicuspid aortic valve (BAV)}

In the SURD-IR, 191 patients (5.6\%) presented with a BAV. However, using SURD valves in BAV is controversial; inserting a SURD prosthesis in a non-circular BAV annulus may result in reduced sealing and paravalvular regurgitation. Nevertheless, during SURD-AVR, unlike TAVI, calcium removal may be effective in reducing paravalvular leaks and promoting a more circular adaptation of the annulus to the valve. It has been suggested that BAV does not represent a contraindication in all cases, but only in patients with BAV type 0 [as described by Sievers et al. (11)] (12). However, available data in these anatomical settings have been limited thus far and robust clinical trials are needed to validate the performance of SURD valves in patients with BAV $(13,14)$. The results of SURD-IR patients with BAV is reported in a focused analysis published in this special issue.

\section{Small aortic annulus}

Some advocate SURD-AVR in patients with a small aortic annulus because of the excellent hemodynamic performance of SURD protheses. When compared with conventional SAVR, SURD-AVR is associated with reduced valve gradients, larger effective orifice areas and lower incidences of patient-prosthesis mismatch $(15,16)$. SURDIR investigators analyzed hemodynamic performance and clinical outcomes in patients with a small ( $\mathrm{S}$ group) versus large (L group) aortic annulus (17). In-hospital results and five-year survival were similar between the two groups. At discharge, mean pressure gradients were $12.8 \pm 5.8$ in the $\mathrm{S}$ group and $14.4 \pm 6.8 \mathrm{mmHg}$ in the $\mathrm{L}$ group $(\mathrm{P}=0.21)$, while indexed effective orifice areas were $0.88 \pm 0.18$ and 
$0.86 \pm 0.22 \mathrm{~cm}^{2} / \mathrm{m}^{2}(\mathrm{P}=0.6)$, respectively. Severe patientprosthesis mismatch occurred in $10.5 \%$ (S group) and $14.3 \%$ ( $\mathrm{L}$ group) of cases $(\mathrm{P}=0.65)$. These findings confirmed that SURD-AVR can be a favorable solution to reduce the risk of patient-prosthesis mismatch in patients with a small aortic annulus.

A subgroup analysis of SURD-IR patients who underwent isolated AVR indicated that valve pressure gradients were significantly lower for the INTUITY valve compared with the Perceval S valve (18). This was also reported in more recent series (19) and may be related to the subannular balloon-expandable stent frame of the INTUITY valve, which could lead to widening of the left ventricular outflow tract and more laminar flow through the prosthesis (20).

\section{Infective endocarditis}

Few patients $(n=43,1 \%)$ with acute valve endocarditis were enrolled in the SURD-IR. Currently, the use of SURD prostheses in the case of endocarditis is still under investigation; SURD-AVR should be performed with caution in such patients with frail and inflammed perivalvular tissue and is contraindicated in cases with annular abscess or destruction (21).

\section{Prosthetic valve choice}

In the SURD-IR, valve prosthesis selection is dependent on surgeon choice and institutional practice. Among 4,636 patients, the Perceval S was implanted in 3,135 $(67.6 \%)$ patients and the EDWARDS INTUITY or INTUITY Elite in 1,501 (32.4\%) patients. Analysis of patient characteristics revealed differences between valve groups. The Perceval S prosthesis was more frequently implanted in high-risk patients compared with the Intuity valve (22). Accordingly, younger patients were more likely to receive an INTUITY valve than a Perceval $\mathrm{S}$ valve. In a series of 1,418 isolated SURD-AVR, $13 \%$ of the INTUITY patients were younger than 65 years compared with $4.6 \%$ of Perceval S patients $(\mathrm{P}<0.001)(18)$. Although this finding reflected real-world surgical practice, it should be interpreted with caution as it is not supported by any evidence. Clinical trials demonstrated excellent mid-term outcomes in both patients receiving Perceval S valves and patients receiving INTUITY valves (23-27); nevertheless, given their recent introduction, no robust data on the durability and performance of these prostheses in the longterm have been reported so far. Thus, we may speculate that SURD-IR surgeons had better long-term expectations in durability from the INTUITY valve (compared with Perceval S), likely based on the assumption that it is of similar durability to the standard Carpentier-Edwards Perimount Magna Ease.

A recent comparative analysis between anterior right thoracotomy (ART) and ministernotomy (MS) access (28) revealed that the Perceval S valve was more frequently applied in the ART group when compared with the INTUITY valve $(41.6 \%$ vs. $18.6 \% ; \mathrm{P}<0.001)$. This is likely favored by (I) the collapsed design of the Perceval $\mathrm{S}$ prosthesis that increases visualization and simplifies valve positioning via limited ART access, and (II) the shortened operative times observed in Perceval S patients. In fact, Perceval S valve implantation demonstrated significantly shorter cross-clamp and CPB times compared with INTUITY valve implantation. In isolated procedures, the reported cross-clamp time is about ten minutes shorter in patients receiving Perceval S, regardless of the surgical approach. However, this was not associated with any differences in clinical outcomes with regard to mortality and postoperative complications $(18,19)$.

\section{Surgical approach}

The treatment of valve pathologies is increasingly focused on developing and popularizing minimally invasive (MI) procedures. When compared with conventional SAVR, minimally invasive aortic valve replacement (MI-AVR) has been associated with reduced postoperative complications, transfusion requirements, length of postoperative stay and increased patient satisfaction (29-32). However, notwithstanding these favorable results, the proportion of patients receiving MI-AVR in daily clinical practice remains disappointingly low, and most SAVR interventions are still performed via a full sternotomy (33). This is mainly due to the perception of increased technical difficulty of MI-AVR that may lead to prolonged operative durations. Because of the simplified and shortened valve implantation process, SURD valves are well suited to facilitate and promote MIAVR. In the SURD-IR study cohort, almost $75 \%$ of isolated AVR were performed through less invasive approaches, with a marked increase to $85.5 \%$ in recent years $(8,18)$. This increased adoption rate of MI-AVR interventions was almost four times as frequent as the observed rate in the German Aortic Valve Registry (GARY) (33). Moreover, as also reported by others (34-36), the increased rate of MIAVR did not translate into considerably prolonged CPB and cross-clamp times using SURD valves. In patients who 
received MI-AVR interventions, the mean $\mathrm{CPB}$ and crossclamp times were 79.4 and 50.2 minutes, respectively (28). These values compared positively with those reported in the multicentric conventional AVR registries, such as the GARY (CPB time 84 minutes, cross-clamp time 60 minutes) and the Society of Thoracic Surgeons (STS) database (CPB time 104.9 minutes, cross-clamp time 77 minutes) $(33,37)$. Thus, by decreasing operative duration and allowing for easier prosthesis implantation via limited access, SURD valves have overcome the main limitations of MI-AVR, and can be considered a primary indication for MI surgery.

Nowadays, two main MI approaches are performed, namely MS and ART. MS incision is the most widely used technique for MI-AVR. The lower prevalence of ART may be attributed to the fact that ART is a longer and more challenging procedure requiring more technical skill. Nevertheless, in the SURD-IR population, the rate of ART (43.6\%) was considerably higher than those reported in previous series $(29,38,39)$. Although the clinical relevance of this observation is debatable, as current evidence shows similar results between patients undergoing ART and MS (32), this strongly supports the assumption that SURD valves facilitate and help promote MI-AVR, regardless of the surgical approach. Recently, SURD-IR investigators compared patients who underwent MS to those who underwent ART and showed that the MS group had a higher risk profile compared with the ART group, and the latter was associated with significant longer operative times (28). Patients who underwent ART showed a decreased rate of postoperative adverse events as well as shorter postoperative lengths of stay. The observed differences between the access groups were likely related to both the higher surgical risk of MS and the advanced experience level of the surgeons performing ART.

The less invasive SURD-AVR also showed promising results in re-operative AVR. Findings from the SURDIR revealed neither conversion to full sternotomy nor mortality, with an acceptable complication rate in 63 patients who underwent redo SURD-AVR through MS or ART (40). A major benefit of less invasive access in redo settings is that adhesion dissection of mediastinal tissue is minimized and, therefore, the risk of bleeding and cardiac or graft injury may be substantially reduced. However, further study is necessary to validate this assumption.

\section{Technical success and operative times}

In large multicenter series, both valves yielded high technical success rates ranging from $95 \%$ to $96.1 \%$ $(26,35,41)$. Evidence from the SURD-IR confirmed this finding, with a successful implantation rate of $97.7 \%$ and no differences between valve types and surgical approaches $(8,18)$. Furthermore, this rate significantly improved over time (from $95.5 \%$ to $98.6 \%$ ) as a result of the growing experience of surgeons and refined surgical techniques. However, it must be mentioned that valve malpositioning emerged as a strong risk factor for in-hospital mortality (odds ratio, $16.2 ; 95 \%$ confidence interval, $2.55-10.8 ; \mathrm{P}=0.003$ ) in patients who underwent isolated, MI SURD-AVR (18). The occurrence of valve malpositioning resulted in considerably longer $\mathrm{CPB}$ and cross-clamp times and a greater incidence of postoperative complications, such as low cardiac output state, respiratory failure and the need for dialysis.

During valve surgery, prolonged CPB and cross-clamp times are well established risk factors for mortality and morbidity (42). Because of the simplified and quicker deployment, SURD valves have been associated with substantially reduced procedural times $(34,43,44)$. In fact, despite a higher rate of MI procedures, the SURD-IR series (45) reported significantly shorter CPB and crossclamp times compared with conventional SAVR series. When compared with the STS database, a time benefit was found both in overall isolated AVR (79 and 51 vs. 106 and $78 \mathrm{~min}$ ) and combined AVR + coronary artery bypass graft (CABG) (106 and $72 v s .147$ and $112 \mathrm{~min}$ ) (45). A clinical advantage determined by shortened operative times with SURD may become more evident in elderly patients, patients carrying serious comorbidities, such as renal insufficiency, peripheral vasculopathy and myocardial dysfunction and patients requiring combined interventions. The latter hypothesis is currently being tested by the SURD-IR investigators.

\section{Results}

\section{In hospital mortality}

Despite the increased risk profile of the SURD-IR study cohort, SURD-AVR yielded excellent clinical outcomes. Overall in-hospital mortality was $2.1 \%$, with $1.3 \%$ and $3.7 \%$ contributed to patients undergoing isolated and combined SURD-AVR, respectively. When stratified according to the risk profile, early mortality was $0.8 \%$ in low-risk (logistic EuroSCORE $<10 \%$ ), isolated SURD-AVR and $2.2 \%$ in increased-risk patients (logistic EuroSCORE $\geq 10 \%$ ) (45). These results compare favorably with those reported in conventional SAVR registries $(6,33,46)$ both in low- and 
high-risk patients.

\section{Stroke}

The occurrence of stroke was $3.2 \%$, which considerably decreased over time, from $4 \%$ to $0.5 \%$ in the isolated SURD-AVR group, and from $4.7 \%$ to $2.4 \%$ in the combined group (8). It is likely that these findings were affected by the reduced patient risk profiles in the last years. However, recent data from the SURD-IR demonstrated that SURD-AVR is associated with satisfactory outcomes in patients of all risk categories (22). On this basis, we speculate that SURD valves, by facilitating less invasive approaches and accelerating the implantation process, provide good immediate results, mainly due to reduced surgical stress to patients; this may be especially true for those in the higher risk category.

\section{Pacemaker implantation}

Concern exists regarding the increased incidence of conduction abnormalities following SURD-AVR (36,47-49). In the SURD-IR, the overall pacemaker implantation rate was $8.9 \%$ with no differences between valve types. However, SURD technologies have demonstrated to be strongly influenced by the "learning curve effect", with improving outcomes over time (45). In the SURD-IR series, a substantial reduction in the pacemaker implantation rate was observed, from $20.6 \%$ to $5.6 \%$ (18). The latter compares satisfactorily with the rate reported after conventional SAVR, and is much lower than those reported after TAVI $(6,33,50,51)$. Simple technical modifications of the SURD valve implantation technique may have contributed to this finding. In particular, a careful avoidance of valve oversizing and low valve positioning is crucial in preventing injuries of conduction tissue. Additionally, the accuracy of decalcification, the traction on the guiding sutures and the balloon pressure may also play a role $(52,53)$. We believe that minimizing post-operative pacemaker implantation rates by optimizing the valve implantation process and identifying proper predictors for conduction disorders are essential to further improve SURD-AVR outcomes.

\section{Valvular leaks}

Although SURD-AVR was associated with low rates of postoperative aortic regurgitation (AR) (severe AR $0.2 \%$, moderate AR $1.2 \%$, mild AR $6.1 \%$ ), these values were still higher than those reported in conventional SAVR interventions (45). However, over the 12 years of SURDIR data collection, both the incidence (from $17.8 \%$ to $2.7 \%$ ) and the severity (severe AR from $0.6 \%$ to 0 , moderate AR from $3.1 \%$ to $1.1 \%$, mild AR from $14.1 \%$ to $1.6 \%$ ) of postoperative AR markedly decreased. This is likely due to the increased surgical experience of the valve implantation technique.

\section{Conclusions}

With more than 4,500 patients enrolled, the SURD-IR is currently the largest independent registry on SURD aortic valves. The SURD-IR provides a real-world picture of SURD-AVR interventions and may refine the decisionmaking process in the search for the most appropriate treatment for patients with aortic valve pathologies. Evidence from the SURD-IR suggests that SURD-AVR is a safe and efficacious alternative to conventional SAVR, with satisfactory and constantly improving outcomes. When compared with SAVR, SURD-AVR allows for shorter CPB and cross-clamp times, promotes MI surgery and provides improved hemodynamic results. Long-term outcomes have yet to be analyzed in order to thoroughly evaluate the performance of these valve technologies over time.

\section{Acknowledgments}

None.

\section{Footnote}

Conflicts of Interest: The authors have no conflicts of interest to declare.

Open Access Statement: This is an Open Access article distributed in accordance with the Creative Commons Attribution-NonCommercial-NoDerivs 4.0 International License (CC BY-NC-ND 4.0), which permits the noncommercial replication and distribution of the article with the strict proviso that no changes or edits are made and the original work is properly cited (including links to both the formal publication through the relevant DOI and the license). See: https://creativecommons.org/licenses/by-nc-nd/4.0/.

\section{References}

1. Nguyen TC, Terwelp MD, Thourani VH, et al. Clinical 
trends in surgical, minimally invasive and transcatheter aortic valve replacement†. Eur J Cardiothorac Surg 2017;51:1086-92.

2. Di Eusanio M, Vessella W, Carozza R, et al. Ultra fasttrack minimally invasive aortic valve replacement: going beyond reduced incisions. Eur J Cardiothorac Surg 2018;53:ii14-8.

3. Magovern GJ, Cromie HW. Sutureless prosthetic heart valves. J Thorac Cardiovasc Surg 1963;46:726-36.

4. Magovern GJ, Liebler GA, Park SB, et al. Twenty-fiveyear review of the Magovern-Cromie sutureless aortic valve. Ann Thorac Surg 1989;48:S33-4.

5. Di Eusanio M, Phan K, Bouchard D, et al. Sutureless Aortic Valve Replacement International Registry (SUAVR-IR): design and rationale from the International Valvular Surgery Study Group (IVSSG). Ann Cardiothorac Surg 2015;4:131-9.

6. Nguyen V, Michel M, Eltchaninoff H, et al. Implementation of transcatheter aortic valve replacement in France. J Am Coll Cardiol 2018;71:1614-27.

7. Gaede L, Blumenstein J, Kim WK, et al. Trends in aortic valve replacement in Germany in 2015: transcatheter versus isolated surgical aortic valve repair. Clin Res Cardiol 2017;106:411-9.

8. Berretta P, Arzt S, Fiore A, et al. Current trends of sutureless and rapid deployment valves: an 11-year experience from the Sutureless and Rapid Deployment International Registry. Eur J Cardiothorac Surg 2020. [Epub ahead of print].

9. Brown JM, O’Brien SM, Wu C, et al. Isolated aortic valve replacement in North America comprising 108,687 patients in 10 years: changes in risks, valve types, and outcomes in the Society of Thoracic Surgeons National Database. J Thorac Cardiovasc Surg 2009;137:82-90.

10. Silaschi M, Conradi L, Treede H, et al. Trends in surgical aortic valve replacement in more than 3,000 consecutive cases in the era of transcatheter aortic valve implantations. Thorac Cardiovasc Surg 2016;64:382-9.

11. Sievers HH, Schmidtke C. A classification system for the bicuspid aortic valve from 304 surgical specimens. J Thorac Cardiovasc Surg 2007;133:1226-33.

12. Gersak B, Fischlein T, Folliguet TA, et al. Sutureless, rapid deployment valves and stented bioprosthesis in aortic valve replacement: recommendations of an International Expert Consensus Panel. Eur J Cardiothorac Surg 2016;49:709-18.

13. Nguyen A, Fortin W, Mazine A, et al. Sutureless aortic valve replacement in patients who have bicuspid aortic valve. J Thorac Cardiovasc Surg 2015;150:851-7.

14. Chiariello GA, Villa E, Messina A, et al. Dislocation of a sutureless prosthesis after type I bicuspid aortic valve replacement. J Thorac Cardiovasc Surg 2018;156:e87-9.

15. Shalabi A, Spiegelstein D, Sternik L, et al. Sutureless versus stented valve in aortic valve replacement in patients with small annulus. Ann Thorac Surg 2016;102:118-22.

16. Borger MA, Dohmen PM, Knosalla C, et al. Haemodynamic benefits of rapid deployment aortic valve replacement via a minimally invasive approach: 1-year results of a prospective multicentre randomized controlled trial. Eur J Cardiothorac Surg 2016;50:713-20.

17. Fiore A, Berretta P, Andreas M, et al. Hemodynamics performance and clinical outcomes after suturless and rapid deployment aortic valve replacements for a small aortic annulus. In: Lisbon: 33rd European Association of Cardiothoracic Surgery Annual Meeting, 2019.

18. Berretta P, Andreas M, Carrel TP, et al. Minimally invasive aortic valve replacement with sutureless and rapid deployment valves: a report from an international registry (Sutureless and Rapid Deployment International Registry)†. Eur J Cardiothorac Surg 2019;56:793-9.

19. D'Onofrio A, Salizzoni S, Filippini C, et al. Surgical aortic valve replacement with new-generation bioprostheses: sutureless versus rapid-deployment. J Thorac Cardiovasc Surg 2019. [Epub ahead of print].

20. Haverich A, Wahlers TC, Borger MA, et al. Threeyear hemodynamic performance, left ventricular mass regression, and prosthetic-patient mismatch after rapid deployment aortic valve replacement in 287 patients. J Thorac Cardiovasc Surg 2014;148:2854-60.

21. Glauber M, Moten SC, Quaini E, et al. International expert consensus on sutureless and rapid deployment valves in aortic valve replacement using minimally invasive approaches. Innovations (Phila) 2016;11:165-73.

22. Santarpino G, Berretta P, Fischlein T, et al. Operative outcome of patients at low, intermediate, high and 'very high' surgical risk undergoing isolated aortic valve replacement with sutureless and rapid deployment prostheses: results of the SURD-IR registry. Eur J Cardiothorac Surg 2019;56:38-43.

23. Shrestha M, Fischlein T, Meuris B, et al. European multicentre experience with the sutureless Perceval valve: clinical and haemodynamic outcomes up to 5 years in over 700 patients. Eur J Cardiothorac Surg 2016;49:234-41.

24. Andreas M, Coti I, Rosenhek R, et al. Intermediateterm outcome of 500 consecutive rapid-deployment surgical aortic valve procedures. Eur J Cardiothorac Surg 
2019;55:527-33.

25. Laufer G, Haverich A, Andreas M, et al. Long-term outcomes of a rapid deployment aortic valve: data up to 5 years. Eur J Cardiothorac Surg 2017;52:281-7.

26. Barnhart GR, Accola KD, Grossi EA, et al. TRANSFORM (Multicenter Experience With Rapid Deployment Edwards INTUITY Valve System for Aortic Valve Replacement) US clinical trial: performance of a rapid deployment aortic valve. J Thorac Cardiovasc Surg 2017;153:241-51.e2.

27. Fischlein T, Meuris B, Hakim-Meibodi K, et al. The sutureless aortic valve at 1 year: A large multicenter cohort study. J Thorac Cardiovasc Surg 2016;151:1617-26.e4.

28. Andreas M, Laufer G, Berretta P, et al. Procedural characteristics and outcome for mini-sternotomy and anterior right thoracotomy with rapid-deployment valves. In: International SFMICS. 20 ISMICS Annual Scientific Meeting. Innovations (Phila) 2019;14:188S. C14.

29. Phan K, Xie A, Di Eusanio M, et al. A meta-analysis of minimally invasive versus conventional sternotomy for aortic valve replacement. Ann Thorac Surg 2014;98:1499-511.

30. Murtuza B, Pepper JR, Stanbridge RD, et al. Minimal access aortic valve replacement: is it worth it? Ann Thorac Surg 2008;85:1121-31.

31. Brown ML, McKellar SH, Sundt TM, et al. Ministernotomy versus conventional sternotomy for aortic valve replacement: a systematic review and meta-analysis. J Thorac Cardiovasc Surg 2009;137:670-9.e5.

32. Phan K, Xie A, Tsai YC, et al. Ministernotomy or minithoracotomy for minimally invasive aortic valve replacement: a Bayesian network meta-analysis. Ann Cardiothorac Surg 2015;4:3-14.

33. Fujita B, Ensminger S, Bauer T, et al. Trends in practice and outcomes from 2011 to 2015 for surgical aortic valve replacement: an update from the German Aortic Valve Registry on 42776 patients. Eur J Cardiothorac Surg 2018;53:552-9.

34. Borger MA, Moustafine V, Conradi L, et al. A randomized multicenter trial of minimally invasive rapid deployment versus conventional full sternotomy aortic valve replacement. Ann Thorac Surg 2015;99:17-25.

35. Laborde F, Fischlein T, Hakim-Meibodi K, et al. Clinical and haemodynamic outcomes in 658 patients receiving the Perceval sutureless aortic valve: early results from a prospective European multicentre study (the Cavalier Trial)†. Eur J Cardiothorac Surg 2016;49:978-86.

36. Andreas M, Wallner S, Habertheuer A, et al. Conventional versus rapid-deployment aortic valve replacement: a single- centre comparison between the Edwards Magna valve and its rapid-deployment successor. Interact Cardiovasc Thorac Surg 2016;22:799-805.

37. Thourani VH, Suri RM, Gunter RL, et al. Contemporary real-world outcomes of surgical aortic valve replacement in 141,905 low-risk, intermediate-risk, and high-risk patients. Ann Thorac Surg 2015;99:55-61.

38. Fattouch K, Moscarelli M, Del Giglio M, et al. Nonsutureless minimally invasive aortic valve replacement: mini-sternotomy versus mini-thoracotomy: a series of 1130 patients. Interact Cardiovasc Thorac Surg 2016;23:253-8.

39. Ghanta RK, Lapar DJ, Kern JA, et al. Minimally invasive aortic valve replacement provides equivalent outcomes at reduced cost compared with conventional aortic valve replacement: a real-world multi-institutional analysis. J Thorac Cardiovasc Surg 2015;149:1060-5.

40. Santarpino G, Berretta P, Kappert U, et al. Minimally invasive redo aortic valve replacement: results from a multicentric registry (SURD-IR). Ann Thorac Surg 2020. [Epub ahead of print].

41. Kocher AA, Laufer G, Haverich A, et al. One-year outcomes of the Surgical Treatment of Aortic Stenosis With a Next Generation Surgical Aortic Valve (TRITON) trial: a prospective multicenter study of rapid-deployment aortic valve replacement with the EDWARDS INTUITY Valve System. J Thorac Cardiovasc Surg 2013;145:110-5; discussion 115-6.

42. Ranucci M, Frigiola A, Menicanti L, et al. Aortic crossclamp time, new prostheses, and outcome in aortic valve replacement. J Heart Valve Dis 2012;21:732-9.

43. Pollari F, Santarpino G, Dell'Aquila AM, et al. Better short-term outcome by using sutureless valves: a propensity-matched score analysis. Ann Thorac Surg 2014;98:611-6; discussion 616.

44. Phan K, Tsai YC, Niranjan N, et al. Sutureless aortic valve replacement: a systematic review and meta-analysis. Ann Cardiothorac Surg 2015;4:100-11.

45. Di Eusanio M, Phan K, Berretta P, et al. Sutureless and Rapid-Deployment Aortic Valve Replacement International Registry (SURD-IR): early results from 3343 patients. Eur J Cardiothorac Surg 2018;54:768-73.

46. Bekeredjian R, Szabo G, Balaban Ü, et al. Patients at low surgical risk as defined by the Society of Thoracic Surgeons Score undergoing isolated interventional or surgical aortic valve implantation: in-hospital data and 1-year results from the German Aortic Valve Registry (GARY). Eur Heart J 2019;40:1323-30.

47. Lazkani M, Yerasi C, Prakash S, et al. Permanent 
pacemaker implantation and paravalvular leak rates following sutureless aortic valve operations. J Card Surg 2018;33:808-17.

48. Ensminger S, Fujita B, Bauer T, et al. Rapid deployment versus conventional bioprosthetic valve replacement for aortic stenosis. J Am Coll Cardiol 2018;71:1417-28.

49. Meco M, Montisci A, Miceli A, et al. Sutureless perceval aortic valve versus conventional stented bioprostheses: meta-analysis of postoperative and midterm results in isolated aortic valve replacement. J Am Heart Assoc 2018;7:e006091.

50. Walther T, Hamm CW, Schuler G, et al. Perioperative results and complications in 15,964 transcatheter aortic

Cite this article as: Di Eusanio M, Berretta P. The sutureless and rapid-deployment aortic valve replacement international registry: lessons learned from more than 4,500 patients. Ann Cardiothorac Surg 2020;9(4):289-297. doi: 10.21037/acs-2020surd-21 valve replacements: prospective data from the GARY registry. J Am Coll Cardiol 2015;65:2173-80.

51. Grover FL, Vemulapalli S, Carroll JD, et al. 2016 Annual Report of The Society of Thoracic Surgeons/American College of Cardiology Transcatheter Valve Therapy Registry. J Am Coll Cardiol 2017;69:1215-30.

52. Yanagawa B, Cruz J, Boisvert L, et al. A simple modification to lower incidence of heart block with sutureless valve implantation. J Thorac Cardiovasc Surg 2016;152:630-2.

53. Fischlein T, Gersak B, Pfeiffer S. How to prevent a pacemaker implantation after sutureless bioprosthesis. J Thorac Cardiovasc Surg 2016;152:635-6. 